\title{
Interaction between Streptococcus pneumoniae and Staphylococcus aureus in paediatric patients suffering from an underlying chronic disease
}

International Journal of Immunopathology and Pharmacology 2015, Vol. 28(4) 497-507 (C) The Author(s) 2015 Reprints and permissions: sagepub.co.uk/journalsPermissions.nav DOI: $10.1177 / 0394632015601486$ iji.sagepub.com (S)AGE

\author{
Susanna Esposito,' Gian Luigi Marseglia,' ${ }^{2}$ Carla Colombo, ${ }^{3}$ \\ Lorenzo lughetti, ${ }^{4}$ Leonardo Terranova,' Valentina lerardi,' \\ Monia Gambino' and Nicola Principi' for the Italian \\ Pneumococcal Study Groups on Asthma, Cystic \\ Fibrosis and Diabetes
}

\begin{abstract}
Little is known about the interaction between Streptococcus pneumoniae and Staphylococcus aureus in school-age children and adolescents suffering from an underlying chronic disease. To increase our knowledge in this regard, an oropharyngeal swab was obtained from school-age children and adolescents suffering from asthma $(n=423)$, cystic fibrosis (CF) $(n=212)$ and type I diabetes mellitus $(D M I)(n=296)$. S. pneumoniae detection and serotyping were performed using a real-time polymerase chain reaction, and $S$. aureus detection was performed using the RIDAGENE MRSA system. Among asthmatic, CF and DMI patients, both pathogens were identified in 65/423 (I5.4\%), 2I/2I2 (9.9\%) and 62/296 (20.9\%) children, respectively; S. pneumoniae alone was identified in 127/434 (30.0\%), $21 / 212(9.9 \%)$ and 86/296 (29.1\%), respectively; S. aureus alone was identified in 58/434 (13.7\%), 78/212 (36.8\%) and 49/296 (16.6\%), respectively. S. pneumoniae colonisation rates were higher in younger children and declined with age, whereas the frequency of $S$. aureus colonisation was quite similar in the different age groups. Among asthmatic and CF patients aged 6-9 years, $S$. aureus carriage was significantly higher in children who were positive for $S$. pneumoniae $(P<0.05)$. No significant association emerged between $S$. aureus carriage and carriage of $S$. pneumoniae serotypes included in the pneumococcal conjugate vaccines (PCVs). This study shows for the first time that school-age children and adolescents with asthma, CF and DMI are frequently colonised by $S$. pneumoniae and $S$. aureus and that no negative relationship seems to exist between these pathogens. Moreover, the supposed protection offered by PCV administration against $S$. aureus colonisation was not demonstrated.
\end{abstract}

\section{Keywords}

pneumococcal colonisation, pneumococcal conjugate vaccine, staphylococcal colonisation, Staphylococcus aureus, Streptococcus pneumoniae

Date received: 10 June 2015; accepted: 27 July 2015

\footnotetext{
'Pediatric Highly Intensive Care Unit, Department of Pathophysiology and Transplantation, Università degli Studi di Milano, Fondazione IRCCS Ca' Granda Ospedale Maggiore Policlinico, Milan, Italy 2Pediatric Clinic, University of Pavia, IRCCS Policlinico "S. Matteo" Foundation, Pavia, Italy

${ }^{3}$ Cystic Fibrosis Center, Lombardia Region, Department of Pathophysiology and Transplantation, Università degli Studi di Milano, Fondazione IRCCS Ca' Granda Ospedale Maggiore Policlinico, Milan, Italy
}

${ }^{4}$ Pediatric Clinic, University of Modena and Reggio Emilia, Modena, Italy

\section{Corresponding author:}

Susanna Esposito, Pediatric Highly Intensive Care Unit, Department of Pathophysiology and Transplantation, Università degli Studi di Milano, Fondazione IRCCS Ca' Granda Ospedale Maggiore Policlinico, Via Commenda 9, 20122 Milano, Italy.

Email: susanna.esposito@unimi.it 


\section{Introduction}

The upper respiratory tract is the reservoir of a diverse community of commensal and potential pathogens. Pharyngeal colonisation is a dynamic process that in a balanced state has a major beneficial role for the human host. ${ }^{1}$ However, an imbalance of the respiratory microbial community can be the basis for developing invasive and mucosal diseases due to an overgrowth of pre-existing pathogens or the acquisition of new infectious agents. Streptococcus pneumoniae and Staphylococcus aureus are common commensals of the upper respiratory tract and among the major causes of bacterial infections in infants and children when imbalance occurs. ${ }^{2,3}$ A better understanding of the incidence of carriage and the relationship between these two pathogens, including their potential for mutual interference, is needed to evaluate the epidemiology of the disease caused by each of them and the impact of related preventative measures, including the pneumococcal conjugate vaccines (PCVs).

A number of studies have investigated the cocolonisation of both healthy and ill children by $S$. pneumoniae and $S$. aureus, but these studies have reported conflicting results. ${ }^{4-8}$ However, most of these studies were carried out in healthy younger children. ${ }^{9}$ Little is known about the interaction between these pathogens in school-age children and adolescents suffering from an underlying chronic disease although in some cases an increased risk of pneumococcal or staphylococcal infection in patients with asthma ${ }^{10}$ or cystic fibrosis $(\mathrm{CF})^{11}$ is well known. Moreover, no data are available on the impact of vaccination with PCVs, given several years before, on combined $S$. pneumoniae and $S$. aureus colonisation of these subjects. To increase our knowledge in this regard, a study specifically designed to evaluate pneumococcal and staphylococcal pharyngeal colonisation rates in school-age children and adolescents suffering from asthma, $\mathrm{CF}$ and type 1 diabetes mellitus (DM1) was carried out.

\section{Materials and methods}

\section{Swab collection}

During the period 1 January 2014 to 30 June 2014, this study enrolled children and adolescents (age range, 6-17 years) suffering from documented asthma, CF and DM1 regularly followed in the outpatient clinic of the University of Milan's DepartmentofPathophysiology and Transplantation and in a number of Department of Pediatrics sited in Pavia, Modena, Verona, Rome and Naples. The protocol was approved by the Ethics Committees of each participating centre. Moreover, written informed consent was obtained from the parent(s) or legal guardian(s) of each study participant and from participants aged older than 8 years. In asthmatic children, the characteristics of asthma were evaluated on the basis of the Global Initiative for Asthma criteria. ${ }^{12}$ Only clinically stable patients with mild to moderate disease were considered suitable for enrolment. Subjects with CF had to be free from acute respiratory exacerbation. Finally, DM1 patients had to be in good metabolic equilibrium. All patients were clinically stable at the time of enrolment; patients with active respiratory infection, those with a chronic underlying disease other than those in the study, and those who had received antibiotic therapy during the previous 2 weeks were excluded. Data regarding the medical history of each subject, including vaccination, were collected by means of a questionnaire that was completed by parents/guardians for children $<15$ years and by the adolescents themselves for those $\geqslant 15$ years. Children were considered fully vaccinated against $S$. pneumoniae if they have received three doses of the heptavalent pneumococcal conjugate vaccine (PCV7) in the first year of life or two doses in the second year or a single dose after the second until the age of 5 years, according to the recommendations of the Italian Ministry of Health (http:// www.salute.gov.it/portale/documentazione/ p6_2_2_1.jsp?lingua $=$ italiano\&id=543).

In each centre, swabbing was carried out by a group of experienced paediatric nurses supervised by a paediatrician. The collection of pharyngeal secretions was performed by means of an oropharyngeal swab because recent studies have suggested that oropharyngeal samples are better for determining $S$. pneumoniae carrier status in schoolage children and adolescents ${ }^{13}$ and may give information regarding $S$. aureus colonisation rates not substantially different from those determined by traditional anterior nares samples. ${ }^{13}$

Oropharyngeal swabs were obtained using the ESwab kit and a polypropylene screw-cap tube with an internal conical shape filled with $1 \mathrm{~mL}$ liquid Amies medium (cat. Number 480CE, Brescia, Copan, Italy). Sampling was carried out using a 
tongue spatula to press the tongue downward to floor of the mouth and swabbing both the tonsillar arches and the posterior pharynx, without touching the sides of the mouth. All swabs were immediately transported to a central laboratory and processed within $2 \mathrm{~h}$ for the identification of $S$. pneumoniae and $S$. aureus.

\section{S.pneumoniae and S. aureus identification}

For $S$. pneumoniae identification, bacterial genomic DNA was directly extracted from the samples using a NucliSENS easyMAG automated extraction system (BioMeriéux, Bagno a Ripoli, Florence, Italy), a $250 \mu \mathrm{L}$ sample input and a generic protocol and was tested for the autolysin-A-encoding gene (lytA) and the wzg (cpsA) gene of $S$. pneumoniae by means of real-time PCR (RT-PCR) as previously described. ${ }^{14}$ Each sample was tested in triplicate and was considered positive if at least two of the three tests revealed the presence of both genes. The level of detection of the test was 16 genome copies. All positive cases were serotyped using primers and probes designed on the basis of the GenBank database sequences (www.ncbi.nlm. nih.gov) of serotypes $1,3,4,5,6 \mathrm{~A}, 6 \mathrm{~B}, 7 \mathrm{~F}, 9 \mathrm{~V}, 14$, $18 \mathrm{C}, 19 \mathrm{~A}, 19 \mathrm{~F}$ and $23 \mathrm{~F}$ (i.e. those in the 13 -valent pneumococcal conjugate vaccine [PCV13]) and synthesised by TIB Molbiol (Genoa, Italy) as previously described. ${ }^{14}$ Analytical specificity was preevaluated by means of computer-aided analyses using Primer-BLAST (www.ncbi.nlm.nih.gov/ tools/primer-blast) and BLAST (www.blast.ncbi. nlm.nih.gov/Blast.cgi) software to compare the sequences with all listed 'bacteria' and 'homo sapiens' sequences.

S. aureus was identified using the RIDAGENE MRSA system (R-Biopharm AG, Darmstadt, Germany). The RIDAGENE MRSA system is a multiplex real-time PCR for the direct, qualitative detection and differentiation of methicillin-resistant $S$. aureus (MRSA) and methicillin-sensitive $S$. aureus (MSSA). An internal control is added to the samples during extraction to determine possible PCR inhibition or DNA extraction failure. After DNA isolation, the mec $\mathrm{A} / m e c \mathrm{C}$ gene, $\mathrm{SCCmec} /$ orf $X$ junction (type I, II, III, IV, V, VI, VII, IX and XI) and the $\operatorname{orf} X$ gene are amplified by TaqMan according to the manufacturer's instructions using the Agilent Stratagene Mx3005P real-time PCR platform. The samples were evaluated as follows: negative, no amplification signal but internal control DNA positive; MRSA, positive for $m e c \mathrm{~A} / m e c \mathrm{C}$, the SCCmec/orf $X$ junction, and orfX; MSSA, positive for both the SCCmec/orf $X$ junction and $\operatorname{orf} X$ or only $\operatorname{orf} X$. The RIDAGENE MRSA system has a limit of detection of $\leqslant 5$ DNA copies per reaction.

\section{Statistical analysis}

A contingency table analysis with the chi-squared or Fisher's exact test, as appropriate, was used to compare differences between groups. For an easier evaluation of carriage modifications in the studied population, this was arbitrarily subdivided into three age groups (6-9 years, $10-14$ years and $\geqslant 15$ years). Subgroup analyses were performed based on age and PCV7 vaccination status. All tests were two-sided, and a $P$ value $<0.05$ was considered statistically significant. Data were analysed using SAS, version 9.2 (SAS Institute, Cary, NC, USA).

\section{Results}

\section{Asthmatic patients}

The study enrolled 423 participants with asthma (70.9\% boys; median age, 10.6 years). Among them $176(41.6 \%)$ were aged between 6 and 9 years, $207(48.9 \%)$ were aged between 10 and 14 years and $40(9.5 \%)$ were aged $\geqslant 15$ years. The carriage of $S$. pneumoniae and $S$. aureus according to age and PCV7 vaccination coverage is shown in Table 1. A total of $173(40.9 \%)$ patients did not carry either $S$. pneumoniae or $S$. aureus. Both pathogens were identified in $65(15.4 \%)$ children, $S$. pneumoniae alone and $S$. aureus alone in 127 $(30.0 \%)$ and $58(13.7 \%)$, respectively. S. pneumoniae colonisation rates were higher in younger children and declined with age $(52.8 \%$ in children aged $6-9$ years, $43.5 \%$ in those aged $10-14$ years and $22.5 \%$ in those aged $\geqslant 15$ years). In contrast, the frequency of $S$. aureus colonisation was quite similar in all age groups $(27.8 \%, 30.4 \%$ and $27.5 \%$ in children aged $6-9$ years, $10-14$ years, and $\geqslant 15$ years, respectively). S. aureus carriage was significantly higher in children who were positive for S. pneumoniae $(65 / 192,33.9 \%$ vs. $58 / 231,25.1 \%$; $P=0.049)$. However, when carriage was evaluated according to the age of enrolled children, it was found that this difference remained statistically significant only for children aged $6-9$ years $(32 / 93$, $34.4 \%$ vs. $17 / 83,20.5 \% ; P=0.04)$. 
Table I. Oropharyngeal carriage of Streptococcus pneumoniae and Staphylococcus aureus in 423 asthmatic children, according to selected subgroups and S. pneumoniae serotypes.

\begin{tabular}{|c|c|c|c|}
\hline \multirow[t]{3}{*}{ S. pneumonia } & \multicolumn{2}{|l|}{ S. aureus } & \multirow[t]{3}{*}{$P$ value } \\
\hline & \multirow{2}{*}{$\frac{\text { Negative }}{\mathrm{n}(\text { row \%) }}$} & \multirow{2}{*}{$\frac{\text { Positive }^{\mathrm{a}}}{\mathrm{n}(\text { row \%) }}$} & \\
\hline & & & \\
\hline \multicolumn{4}{|l|}{ All subjects } \\
\hline Negative & $173(74.9)$ & $58(25.1)$ & \\
\hline Positive & $127(66.1)$ & $65(33.9)$ & 0.049 \\
\hline \multicolumn{4}{|l|}{ Age subgroups } \\
\hline \multicolumn{4}{|l|}{$6-9$ years $(n=176)$} \\
\hline Negative & $66(79.5)$ & $17(20.5)$ & \\
\hline Positive & $61(65.6)$ & $32(34.4)$ & 0.04 \\
\hline \multicolumn{4}{|l|}{$10-14$ years $(n=207)$} \\
\hline Negative & $85(72.6)$ & $32(27.4)$ & \\
\hline Positive & $59(65.6)$ & $31(34.4)$ & 0.27 \\
\hline \multicolumn{4}{|l|}{$15-17$ years $(n=40)$} \\
\hline Negative & $22(71.0)$ & $9(29.0)$ & \\
\hline Positive & 7 (77.8) & $2(22.2)$ & 0.99 \\
\hline \multicolumn{4}{|l|}{ Vaccination with PCV7 } \\
\hline \multicolumn{4}{|l|}{ Unvaccinated $(n=228)$} \\
\hline Negative & $93(72.1)$ & $36(27.9)$ & \\
\hline Positive & $67(67.7)$ & $32(32.3)$ & 0.47 \\
\hline \multicolumn{4}{|l|}{ Vaccinated $(n=195)$} \\
\hline Negative & $80(78.4)$ & $22(21.6)$ & \\
\hline Positive & $60(64.5)$ & $33(35.5)$ & 0.03 \\
\hline \multicolumn{4}{|l|}{ Serotypes of PCV7b } \\
\hline Positive for any PCV7 serotype & $119(66.5)$ & $60(33.5)$ & \\
\hline $\begin{array}{l}\text { Negative for PCV7 serotypes, } \\
\text { but positive for S. pneumonia }\end{array}$ & $8(6 \mid .5)$ & $5(38.5)$ & 0.77 \\
\hline \multicolumn{4}{|l|}{ Serotypes of PCVI $3^{b}$} \\
\hline Positive for any PCVI 3 serotype & $119(65.4)$ & $63(34.6)$ & \\
\hline $\begin{array}{l}\text { Negative for PCVI } 3 \text { serotypes, } \\
\text { but positive for S. pneumonia }\end{array}$ & $8(80.0)$ & $2(20.0)$ & 0.50 \\
\hline \multicolumn{4}{|l|}{ Specific serotypes ${ }^{c}$} \\
\hline Negative for serotype 3 & $283(70.7)$ & $117(29.3)$ & \\
\hline Positive for serotype 3 & 17 (73.9) & $6(26.1)$ & 0.75 \\
\hline Negative for serotype 4 & $255(7 I .2)$ & $103(28.8)$ & \\
\hline Positive for serotype 4 & $45(69.2)$ & $20(30.8)$ & 0.74 \\
\hline Negative for serotype 5 & 277 (7I.9) & $108(28.1)$ & \\
\hline Positive for serotype 5 & $23(60.5)$ & $15(39.5)$ & 0.14 \\
\hline Negative for serotype 6A & $296(71.8)$ & $116(28.2)$ & \\
\hline Positive for serotype $6 \mathrm{~A}$ & $4(36.4)$ & $7(63.6)$ & 0.02 \\
\hline Negative for serotype 9V & $274(7 I .5)$ & $109(28.5)$ & \\
\hline Positive for serotype 9V & $26(65.0)$ & $14(35.0)$ & 0.39 \\
\hline Negative for serotype 19A & $296(71.7)$ & $117(28.3)$ & \\
\hline Positive for serotype I9A & $4(40.0)$ & $6(60.0)$ & 0.07 \\
\hline Negative for serotype I9F & $189(73.8)$ & $67(26.2)$ & \\
\hline Positive for serotype I9F & III (66.5) & $56(33.5)$ & 0.10 \\
\hline
\end{tabular}

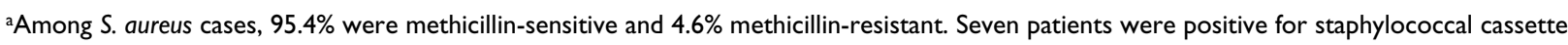
chromosome mec.

${ }^{\mathrm{b} A m o n g} 192$ subjects positive for $S$. pneumoniae.

cOnly serotypes of $S$. pneumoniae for which at least 10 subjects were positive are presented in the table.

PCV7, 7-valent pneumococcal conjugate vaccine; PCVI3, I3-valent pneumococcal conjugate vaccine. 
Among vaccinated subjects, $S$. aureus was identified more frequently in children who were positive for $S$. pneumoniae than in those who were negative $(33 / 93,35.5 \%$ vs. $22 / 102,21.6 \% ; P=$ $0.03)$. In contrast, among unvaccinated subjects, the percentage of those colonised by $S$. aureus was not materially different in subjects positive and negative for $S$. pneumoniae $(32 / 99,32.3 \%$ vs. $36 / 129,27.9 \%, P=0.47)$. Among 192 subjects positive for $S$. pneumoniae, no significant association emerged between $S$. aureus carriage and carriage of $S$. pneumoniae serotypes included in PCV7 $(P=0.77)$ nor PCV13 $(P=0.50)$.

\section{CF patients}

A total of 212 CF patients were enrolled (48.1\% boys; median age, 11.7 years). Among them, 63 $(29.7 \%)$ were aged $6-9$ years, $102(48.1 \%)$ were aged $10-14$ years and $47(22.2 \%)$ were aged $\geqslant 15$ years. The carriage of $S$. pneumoniae and $S$. aureus according to age and PCV7 vaccination coverage is shown in Table 2. A great number of patients were not colonised by the studied pathogens $(92,43.4 \%)$. Both pathogens were identified in $21(9.9 \%)$ children, $S$. pneumoniae alone and $S$. aureus alone in $21(9.9 \%)$ and $78(36.8 \%)$, respectively. S. pneumoniae colonisation rates were higher in younger children and declined with age (28.6\% in children aged 6-9 years, $17.6 \%$ in those aged $10-14$ years and $12.8 \%$ in those aged $\geqslant 15$ years). The frequency of $S$. aureus colonisation was slightly higher in children aged 10-14 years $(51 / 102,50.0 \%)$ in comparison with patients aged $6-9$ years and $\geqslant 15$ years $(29 / 63,46.0 \%$ and $19 / 47,40.4 \%$, respectively). S. aureus carriage was quite similar in children positive and negative for $S$. pneumoniae $(21 / 42,50.0 \%$ vs. $78 / 170$, $45.9 \% ; P=0.63)$. However, in younger children, colonisation by $S$. aureus was significantly higher in patients positive for $S$. pneumoniae than in those who were negative $(12 / 18,66.6 \%$ vs. $17 / 45$, $37.8 \% ; P=0.04)$.

Among vaccinated subjects, $S$. aureus was identified with similar frequency in children who were positive and negative for $S$. pneumoniae (3/10, $30.0 \%$ vs. $10 / 25,40.0 \% ; P=0.71)$. Similarly, among unvaccinated subjects, the percentage of those colonised by $S$. aureus was not materially different in subjects positive and negative for
S. pneumoniae $(18 / 32,56.3 \%$ vs. $68 / 145,46.9 \%$; $P=0.34)$.

Among 42 subjects positive for $S$. pneumoniae, no significant association emerged between $S$. aureus carriage and carriage of the PCV7 and PCV13 S. pneumoniae serotypes, although the number of subjects positive for serotypes other than PCV7 was small $(n=3)$.

\section{Diabetic patients}

A total of 296 DM1 patients were enrolled (51.3\% boys; median age, 12.7 years). Among them, 61 (20.6\%) were aged 6-9 years, $154(52.0 \%)$ were aged $10-14$ years and $81(27.4 \%)$ were aged $\geqslant 15$ years. The carriage of $S$. pneumoniae and $S$. aureus according to age and PCV7 vaccination coverage is shown in Table 3. Approximately one-third of the patients were not colonised by the studied bacteria $(99,33.4 \%)$. Both pathogens were identified in $62(20.9 \%)$ children, and S. pneumoniae alone and $S$. aureus alone were identified in $86(29.1 \%)$ and 49 (16.6\%), respectively. S. pneumoniae colonisation rates were higher in younger children and declined with age $(62.3 \%$ in children aged 6-9 years, $53.9 \%$ in those aged $10-14$ years and $33.3 \%$ in those aged $\geqslant 15$ years). The frequency of $S$. aureus colonisation was quite similar in the three age groups even if slightly higher in children aged 6-9 years $(25 / 61,41.0 \%)$ in comparison with patients aged $10-14$ years and $\geqslant 15$ years $(56 / 154$, $36.4 \%$ and $30 / 81,37.0 \%$, respectively). S. aureus carriage was not significantly different between children positive and negative for $S$. pneumoniae (62/148, $41.9 \%$ vs. $49 / 148,33.1 \% ; P=0.12)$, even when different age groups were considered.

Among vaccinated subjects, $S$. aureus was identified more frequently in children who were positive for $S$. pneumoniae than those who were negative (26/56, $46.4 \%$ vs. $14 / 53,26.4 \% ; P=0.03)$. In contrast, among unvaccinated subjects, the percentage of those colonised by $S$. aureus was similar in subjects positive and negative for $S$. pneumoniae $(35 / 88$, $39.8 \%$ vs. $32 / 86,37.2 \% ; P=0.73$ ).

Among 148 subjects positive for $S$. pneumoniae, no significant association emerged between $S$. aureus carriage and carriage of the PCV7 and PCV13 S. pneumoniae serotypes, although the number of subjects positive for serotypes other than PCV7 was small $(n=4)$. 
Table 2. Oropharyngeal carriage of Streptococcus pneumoniae and Staphylococcus aureus in 212 children with cystic fibrosis, also according to selected subgroups and Streptococcus pneumoniae serotypes.

\begin{tabular}{|c|c|c|c|}
\hline \multirow[t]{3}{*}{ S. pneumoniae } & \multicolumn{2}{|l|}{ S. aureus } & \multirow[t]{3}{*}{$P$ value } \\
\hline & \multirow{2}{*}{$\frac{\text { Negative }}{\text { n (row \%) }}$} & \multirow{2}{*}{$\frac{\text { Positive }^{\mathrm{a}}}{\mathrm{n} \text { (row \%) }}$} & \\
\hline & & & \\
\hline \multicolumn{4}{|l|}{ All subjects } \\
\hline Negative & $92(54.1)$ & $78(45.9)$ & \\
\hline Positive & $21(50.0)$ & $21(50.0)$ & 0.63 \\
\hline \multicolumn{4}{|l|}{ Age subgroups } \\
\hline \multicolumn{4}{|l|}{$6-9$ years $(n=63)$} \\
\hline Negative & $28(62.2)$ & $17(37.8)$ & \\
\hline Positive & $6(33.3)$ & $12(66.7)$ & 0.04 \\
\hline \multicolumn{4}{|l|}{$10-14$ years $(n=102)$} \\
\hline Negative & $40(47.6)$ & $44(52.4)$ & \\
\hline Positive & II (6I.I) & 7 (38.9) & 0.30 \\
\hline \multicolumn{4}{|l|}{$15-17$ years $(n=47)$} \\
\hline Negative & $24(58.5)$ & $17(4 \mid .5)$ & \\
\hline Positive & $4(66.7)$ & $2(33.3)$ & 0.99 \\
\hline \multicolumn{4}{|l|}{ Vaccination with PCV7 } \\
\hline \multicolumn{4}{|l|}{ Unvaccinated $(\mathrm{n}=177)$} \\
\hline Negative & $77(53.1)$ & $68(46.9)$ & \\
\hline Positive & $14(43.7)$ & $18(56.3)$ & 0.34 \\
\hline \multicolumn{4}{|l|}{ Vaccinated $(n=35)$} \\
\hline Negative & $15(60.0)$ & $10(40.0)$ & \\
\hline Positive & $7(70.0)$ & $3(30.0)$ & 0.71 \\
\hline \multicolumn{4}{|l|}{ Serotypes of PCV $7^{b}$} \\
\hline Positive for any PCV7 serotype & $20(5 \mathrm{I} .3)$ & $19(48.7)$ & \\
\hline $\begin{array}{l}\text { Negative for PCV7 serotypes, but } \\
\text { positive for S. pneumonia }\end{array}$ & I (33.3) & $2(66.7)$ & 0.99 \\
\hline \multicolumn{4}{|l|}{ Serotypes of PCVI $3^{b}$} \\
\hline Positive for any PCVI 3 serotype & $20(50.0)$ & $20(50.0)$ & \\
\hline $\begin{array}{l}\text { Negative for PCVI } 3 \text { serotypes, } \\
\text { but positive for S. pneumonia }\end{array}$ & I (50.0) & I (50.0) & 0.99 \\
\hline \multicolumn{4}{|l|}{ Specific serotypesc } \\
\hline Negative for serotype 5 & $104(5 \mid .5)$ & $98(48.5)$ & \\
\hline Positive for serotype 5 & $9(90.0)$ & I (I0.0) & 0.02 \\
\hline Negative for serotype I9F & $95(53.7)$ & $82(46.3)$ & \\
\hline Positive for serotype I9F & $18(51.4)$ & $17(48.6)$ & 0.81 \\
\hline
\end{tabular}

${ }^{a}$ Among S. aureus cases, $91.9 \%$ were methicillin-sensitive and $8.1 \%$ methicillin-resistant. Six patients were positive for staphylococcal cassette chromosome mec.

${ }^{\text {bAmong }} 42$ subjects positive for $S$. pneumoniae.

'Only serotypes of $S$. pneumoniae for which at least 10 subjects were positive are presented in the Table.

PCV7, 7-valent pneumococcal conjugate vaccine; PCVI3, I3-valent pneumococcal conjugate vaccine.

\section{Discussion}

Whether bacteria can colonize or not is determined by many ecological factors including the availability of resources (i.e. nutrients, space, attachment space), host immune responses and the presence of toxins or harmful substances. ${ }^{15}$ Moreover, the interference between bacterial resident populations producing harmful substances (i.e. bacterocins) $)^{15,16}$ or inducing an immune response ${ }^{17,18}$ can play a role at this regard. In the case of $S$. pneumoniae and $S$. aureus, epidemiological studies showed that co-colonisation is rarer than expected and suggested that $S$. pneumoniae colonisation is reduced in presence of $S$. pneumoniae. ${ }^{19,20}$ Knowledge of carriage characteristics of these pathogens, which is important in healthy children, is even more relevant in subjects with chronic underlying disease because applicable 
Table 3. Oropharyngeal carriage of Streptococcus pneumoniae and Staphylococcus aureus in 296 children with type I diabetes mellitus, also according to selected subgroups and S. pneumoniae serotypes.

\begin{tabular}{|c|c|c|c|}
\hline \multirow[t]{3}{*}{ S. pneumoniae } & \multicolumn{2}{|l|}{ S. aureus } & \multirow[t]{3}{*}{$P$ value } \\
\hline & \multirow{2}{*}{$\frac{\text { Negative }}{\mathrm{n} \text { (row \%) }}$} & \multirow{2}{*}{$\frac{\text { Positive }^{a}}{\text { n (row \%) }}$} & \\
\hline & & & \\
\hline \multicolumn{4}{|l|}{ All subjects } \\
\hline Negative & 99 (66.9) & $49(33.1)$ & \\
\hline Positive & $86(58.1)$ & $62(41.9)$ & 0.12 \\
\hline \multicolumn{4}{|l|}{ Age subgroups } \\
\hline \multicolumn{4}{|l|}{$6-9$ years $(n=61)$} \\
\hline Negative & $15(65.2)$ & $8(34.8)$ & \\
\hline Positive & $21(55.3)$ & $17(44.7)$ & 0.44 \\
\hline \multicolumn{4}{|l|}{$10-14$ years $(n=154)$} \\
\hline Negative & $5 I(7 I .8)$ & $20(28.2)$ & \\
\hline Positive & $47(56.6)$ & $36(43.4)$ & 0.051 \\
\hline \multicolumn{4}{|l|}{$15-17$ years $(n=81)$} \\
\hline Negative & $33(6 I . I)$ & $21(38.9)$ & \\
\hline Positive & $18(66.7)$ & $9(33.3)$ & 0.63 \\
\hline \multicolumn{4}{|l|}{ Vaccination with $P C V 7^{\mathrm{b}}$} \\
\hline \multicolumn{4}{|l|}{ Unvaccinated $(n=174)$} \\
\hline Negative & $54(62.8)$ & $32(37.2)$ & \\
\hline Positive & $53(60.2)$ & $35(39.8)$ & 0.73 \\
\hline \multicolumn{4}{|l|}{ Vaccinated $(\mathrm{n}=109)$} \\
\hline Negative & $39(73.6)$ & $14(26.4)$ & \\
\hline Positive & $30(53.6)$ & $26(46.4)$ & 0.03 \\
\hline \multicolumn{4}{|l|}{ Serotypes of PCV7c } \\
\hline Positive for any PCV7 serotype & $83(57.6)$ & $61(42.4)$ & \\
\hline $\begin{array}{l}\text { Negative for PCV7 serotypes, } \\
\text { but positive for } \mathrm{S} \text {. pneumoniae }\end{array}$ & $3(75.0)$ & I (25.0) & 0.64 \\
\hline \multicolumn{4}{|l|}{ Serotypes of PCVI $3^{c}$} \\
\hline Positive for any PCVI 3 serotype & $83(57.2)$ & $62(42.8)$ & \\
\hline $\begin{array}{l}\text { Negative for PCVI } 3 \text { serotypes, } \\
\text { but positive for S. pneumoniae }\end{array}$ & $3(100.0)$ & $0(0.0)$ & 0.26 \\
\hline \multicolumn{4}{|l|}{ Specific serotypes $^{\mathrm{d}}$} \\
\hline Negative for serotype 4 & $166(64.3)$ & $92(35.7)$ & \\
\hline Positive for serotype 4 & $19(50.0)$ & $19(50.0)$ & 0.09 \\
\hline Negative for serotype 5 & $168(62.7)$ & $100(37.3)$ & \\
\hline Positive for serotype 5 & 17 (60.7) & II (39.3) & 0.84 \\
\hline Negative for serotype 9V & $153(6 \mid .7)$ & $95(38.3)$ & \\
\hline Positive for serotype 9V & $32(66.7)$ & $16(33.3)$ & 0.51 \\
\hline Negative for serotype I9A & $176(62.2)$ & $107(37.8)$ & \\
\hline Positive for serotype I9A & $9(69.2)$ & $4(30.8)$ & 0.77 \\
\hline Negative for serotype I9F & $104(66.7)$ & $52(33.3)$ & \\
\hline Positive for serotype I9F & $81(57.9)$ & $59(42.1)$ & 0.12 \\
\hline
\end{tabular}

aAmong S. aureus cases, $97.3 \%$ were methicillin-sensitive and $2.7 \%$ methicillin-resistant. Five patients were positive for staphylococcal cassette chromosome mec.

bThirteen subjects were excluded due to missing information on PCV7 status.

cAmong I 48 subjects positive for S. pneumoniae.

dOnly serotypes of $S$. pneumoniae for which at least 10 subjects were positive are presented in the Table.

PCV7, 7-valent pneumococcal conjugate vaccine; PCVI3, 13-valent pneumococcal conjugate vaccine.

information can led to more appropriate prophylactic and therapeutic measures. Unfortunately, $S$. pneumoniae and $S$. aureus carriage and reciprocal interactions in children with severe chronic underlying disease have received poor attention in recent years. Only HIV-infected children have been studied with conflicting results. In a first cross-sectional study, it was indicated that no 
association between pneumococcal and $S$. aureus colonisation could be demonstrated. ${ }^{21}$ More recently, a longitudinal study found that a lower prevalence of pneumococcal colonisation could lead to an increase in $S$. aureus presence in nasopharyngeal niche. ${ }^{22}$ This study adds some new information regarding older children with diseases, such as asthma, CF and DM1, for whom several studies have evidenced an increased risk of development of both pneumococcal and staphylococcal infections and for whom, at least in some cases, colonisation with these pathogens was expected to have a role in favouring the development of disease itself or its worsening.

Asthmatic patients, independent of asthma severity, suffer from pneumococcal diseases more frequently then healthy subjects. ${ }^{10}$ Pneumococcal colonisation in the neonatal period is associated with frequent wheezing episodes in later paediatric life. ${ }^{22}$ Finally, $S$. aureus colonisation is more common in asthmatic patients ${ }^{23}$ and is associated with wheeze and asthma, ${ }^{24}$ possibly through the bacterial enterotoxin sensitisation..$^{25}$

In CF patients, $S$. aureus is a common coloniser of the respiratory tract and one of the most common pathogens responsible of the infective exacerbations and progressive decline of lung function. ${ }^{11}$ Moreover, it has been recently demonstrated that children with this disease are frequently carriers of S. pneumoniae, ${ }^{2}$ and $\mathrm{CF}$ samples can have peculiar characteristics that can lead to increased virulence and significant lung damage. ${ }^{2}$ Colonising pneumococci form well-organised biofilm communities in the nasopharyngeal environment, with bacteria that are resistant to commonly prescribed antipneumococcal antibiotics. Recent studies have shown that changes in the nasopharyngeal environment caused by concomitant virus infection, modifications in the microflora, inflammation or other host assaults trigger active release of pneumococci from biofilms. ${ }^{26,27}$ These dispersed bacteria have distinct phenotypic properties and transcriptional profiles different from both biofilm and brothgrown, planktonic bacteria, resulting in a significantly increased virulence in vivo. ${ }^{27}$

The higher risk of pneumococcal infections, including IPD, in DM1 patients compared with healthy subjects has been well known for many years and was recently confirmed by the observation that, despite the introduction of vaccine prophylaxis, it remained three times higher in the UK than in the general population. ${ }^{28-30}$ Finally, the incidence of staphylococcal infections in DM1 patients is not marginal and some of them can be particularly severe and difficult to treat. ${ }^{31}$

Regarding pneumococcal colonisation, this study shows that in all the study groups it decreases with age. This is not surprising because a decline of pneumococcal colonisation from infancy to adolescence has been repeatedly reported in healthy children and mainly ascribed to the maturation of the immune system. ${ }^{8,32}$ However, the incidence of colonisation in the studied children was significantly higher than that reported in other studies involving younger children. ${ }^{32}$ Host immune characteristics and the greater sensitivity of the molecular methods used in this study in comparison with cultural methods used in most of the studies carried out in healthy children could explain this finding. However, this finding deserves attention because it supports the use of prophylactic measures against pneumococcal infections for school-age children and adolescents with asthma, CF and DM1.33

Previous PCV7 vaccination did not influence either absolute pneumococcal carriage or carriage of pneumococcal serotypes included in the conjugate vaccine or in PCV13. Other studies have evidenced that PCV administration has a relevant impact on colonisation, significantly reducing carriage of serotypes included in the administered vaccine. ${ }^{34}$ However, these studies were carried out only a few months after vaccine use and the waning of vaccine-induced immunity with time could explain the finding, as already suggested by the data recently collected in healthy older children and adolescents. ${ }^{35}$ On the other hand, this could be a problem because the waning of immunity against carriage could favour the development of diseases due to the initial elimination of the same serotypes and suggests the use of further booster vaccine doses to maintain protection. ${ }^{33}$

Regarding $S$. aureus colonisation, it cannot be forgotten that, from a theoretical point of view, the presence of $S$. pneumoniae in the pharynx should reduce $S$. aureus carriage. $S$. pneumoniae produces hydrogen peroxide that inhibits $S$. aureus $^{36}$ and can further reduce staphylococcal carriage through immune mediated mechanisms based on cross-reactive antibodies against common conserved dehydrogenases. ${ }^{37}$ Moreover, the expression of phosphorylcholine and neuraminidase production by $S$. pneumoniae may contribute 
to competitive effects between these bacterial species. ${ }^{38}$ Finally, pneumococci containing pilusislands have been negatively associated with $S$. aureus colonisation. ${ }^{39}$

However, in this study in which the incidence of $S$. aureus colonisation was in line with what was previously reported in healthy older children, ${ }^{13}$ pneumococcal colonisation was not associated with a reduced $S$. aureus colonisation. In asthmatic children and in those with CF aged 6-9 years, pharyngeal positivity for $S$. pneumoniae was associated with an increase in $S$. aureus colonisation. In children with DM1, the incidence of $S$. aureus carriage was similar in patients with or without pneumococcal colonisation. Moreover, no impact of PCV7 administration was evidenced in any of the groups. These findings are in contrast with those reported in healthy subjects who displayed a negative relationship between $S$. pneumoniae and $S$. aureus and a positive effect of PCV7 administration in conditioning $S$. aureus carriage. ${ }^{35}$ The lack of a negative interaction between the two pathogens is difficult to explain. Bacterial colonisation with a single infectious agent depends on several factors. Host defences, environmental factors, and carriage of other bacteria and/or viruses may play a role. The importance of direct bacterial effectors, viralinduced bacterial adhesion, viral-derived disruption of the respiratory epithelium, production of viral products, and interference with the host immune system in conditioning type and degree of colonisation has been demonstrated. ${ }^{38}$ All of these factors were not evaluated, and it is possible that their role could have influenced $S$. pneumoniae and $S$. aureus colonisation of the studied children. The interference of other unknown factors in conditioning carriage is also supported by the data regarding PCV7 administration and $S$. aureus colonisation, which contrast with the data from in healthy younger children. Previous studies have found that younger children who were colonised by primarily PCV7 serotypes were less frequent carriers of $S$. aureus compared with subjects colonised with other serotypes, ${ }^{9}$ suggesting that the serotypes included in PCV7 could protect against $S$. aureus colonisation. In this study, children who had received PCV7 several years before were colonised by the same serotypes included in the vaccine, and despite this vaccination, they were frequently colonised by $S$. aureus. The waning of immune protection against pneumococcal colonisation some years after the last vaccine dose has been already demonstrated in healthy children ${ }^{35}$ and can be the cause of the persistence of carriage of PCV7 serotypes in the children enrolled in this study despite previous vaccination. Finally, with very few exceptions, $S$. aureus was not associated with any of the serotypes included in both PCV7 and PCV13, confirming that in this study $S$. pneumoniae and $S$ aureus colonisation were completely independent.

In conclusion, this study shows for the first time that school-age children and adolescents with asthma, CF and DM1 are frequently colonised by $S$. pneumoniae and $S$. aureus and that no negative relationship seems to exist between these pathogens. It is not known whether this colonisation depends on the immune characteristics and the infectious history of these patients or from other previously unidentified factors. Moreover, the supposed protection offered by PCV7 administration against $S$. aureus colonisation was not demonstrated and no substantial association between single pneumococcal serotypes and $S$. aureus was evidenced. Further studies are needed to clarify the relationships between bacterial pathogens and the role of $\mathrm{PCV}$ in conditioning staphylococcal colonisation.

\section{Acknowledgements}

We would like to thank all participants in the Italian Pneumococcal Study Groups on Asthma, Cystic Fibrosis and Diabetes: Susanna Esposito, Nicola Principi, Maria Francesca Patria, Claudia Tagliabue, Mara Lelii, Luca Ruggiero, Leonardo Terranova, Alberto Zampiero, Valentina Montinaro, Valentina Ierardi, Monia Gambino (Pediatric Highly Intensive Care Unit, Department of Pathophysiology and Transplantation, Università degli Studi di Milano, Fondazione IRCCS Ca' Granda Ospedale Maggiore Policlinico, Milan, Italy); Gianluigi Marseglia, Amelia Licari, Valentina Trovamala (Pediatric Clinic, University of Pavia, IRCCS Policlinico "S. Matteo" Foundation, Pavia, Italy); Michele Miraglia del Giudice, Annalisa Allegorico (Pediatric Clinic, II Naples University, Naples, Italy); Alessandro Bodini (Pediatric Clinic, University of Verona, Verona, Italy); Alberto Martelli (Pediatric Unit, Garbagnate Hospital, Garbagnate, Italy); Eugenio Baraldi (Pediatric Clinic, University of Padua, Padua, Italy); Alessandro Fiocchi, Oscar Mazzina (Allergology Unit, IRCCS Bambino Gesù Hospital, Rome, Italy); Carla Colombo, Arianna Bisogno, Fabiola Corti, Rosa Moresco (Cystic Fibrosis Center, Lombardia Region, Department of Pathophysiology and Transplantation, Università degli Studi di Milano, Fondazione IRCCS Ca' Granda Ospedale Maggiore Policlinico, Milan, Italy); Valeria Raia, Antonella Tosco, Federica Impronta (Cystic 
Fibrosis Center, Campania Region, Department of Pediatrics, University of Naples Federico II, Naples, Italy); Vincenzina Lucidi, Enza Montemitro (Cystic Fibrosis Center, Lazio Region, IRCCS Bambino Gesù Hospital, Rome, Italy); Sonia Volpi, Marianna Passiu, Ilaria Meneghelli (Cystic Fibrosis Center, Veneto Region, University and Hospital Trust of Verona, Verona, Italy); Lorenzo Iughetti, Viviana Patianna (Pediatric Clinic, University of Modena and Reggio Emilia, Modena, Italy); Marco Cappa, Maria Cristina Matteoli, Patrizia Patera, Paolo Ciampalini, Riccardo Schiaffini, (Unit of Endocrinology and Diabetic Bambino Gesù Children's Hospital IRCCS, Rome, Italy); Claudio Maffeis, Marco Marigliano, Anita Morandi (Regional Centre for Pediatric Diabetes, Clinical Nutrition and Obesity, ULSS 20, and University of Verona, Verona, Italy); Franco Chiarelli, Paola Cipriano (Department of Pediatrics, University of Cheti, Chieti, Italy); Gianni Bona, Silvia Parlamento, Erica Pozzi (Division of Pediatrics, Department of Health Sciences, Università del Piemonte Orientale "Amedeo Avogadro", Novara, Italy).

\section{Declaration of conflicting interests}

The author(s) declared no potential conflicts of interest with respect to the research, authorship, and/or publication of this article.

\section{Funding}

This study was supported by a grant from the Italian Ministry of Health (Bando Giovani Ricercatori 2009) and an unrestricted educational grant from Pfizer International to the Italian Society for Pediatric Infectious Diseases (SITIP).

\section{References}

1. Blaser MJ and Falkow S (2009) What are the consequences of the disappearing human microbiota? Nature Reviews Microbiology 7: 887-894.

2. Simell B, Auranen K, Käyhty H, et al. (2012) The fundamental link between pneumococcal carriage and disease. Expert Review of Vaccines 11: 841-855.

3. Wertheim HFL, Melles DC, Vos MC, et al. (2005) The role of nasal carriage in Staphylococcus aureus infections. Lancet Infectious Diseases 5: 751-762.

4. Cohen R, Levy C, ThollotF, et al. (2007) Pneumococcal conjugate vaccine does not influence Staphylococcus aureus carriage in young children with acute otitis media. Clinical Infectious Diseases 45: 1583-1587.

5. Quintero B, Araque M, van der Gaast-de Jongh C, et al. (2011) Epidemiology of Streptococcus pneumoniae and Staphylococcus aureus colonization in healthy Venezuelan children. European Journal of Clinical Microbiology \& Infectious Diseases 30: 7-19.
6. Regev-Yochay G, Dagan R, Raz M, et al. (2004) Association between carriage of Streptococcus pneumoniae and Staphylococcus aureus in children. Journal of the American Medical Association 292: 716-720.

7. McNally LM, Jeena PM, Gajee K, et al. (2006) Lack of association between the nasopharyngeal carriage of Streptococcus pneumoniae and Staphylococcus aureus in HIV-1-infected South African children. Journal of Infectious Diseases 194: 385-390.

8. Kwambana BA, Barer MR, Bottomley C, et al. (2011) Early acquisition and high nasopharyngeal co-colonisation by Streptococcus pneumoniae and three respiratory pathogens amongst Gambian newborns and infants. BMC Infectious Diseases 11: 175.

9. Xu Q, Almudervar A, Casey JR, et al. (2012) Nasopharyngeal bacterial interactions in children. Emerging Infectious Diseases 18: 1738-1745.

10. Esposito S, Musio A and Principi N (2013) Paediatric asthma and pneumococcal vaccination. Vaccine 31: 5015-5019.

11. Mogayzel PJ Jr, Naureckas ET, Robinson KA, et al. (2013) Cystic fibrosis pulmonary guidelines. Chronic medications for maintenance of lung health. American Journal of Respiratory and Critical Care Medicine 187: 680-689.

12. Global Initiative for Asthma. Global strategy for asthma management and prevention 2014. Available at: www.ginasthma.com (accessed 22nd July 2015).

13. Principi N, Terranova L, Zampiero A, et al. (2014) Oropharyngeal and nasopharyngeal sampling for the detection of adolescent Streptococcus pneumoniae carriers. Journal of Medical Microbiology 63: 393-398.

14. Esposito S, Terranova L, Zampiero A, et al. (2014) Oropharyngeal and nasal Staphylococcus aureus carriage by healthy children. BMC Infectious Diseases 14: 3844 .

15. Chao L and Levin BR (1981) Structured habitats and the evolution of anticompetitor toxins in bacteria. Proceedings of the National Academy of Sciences of the United States of America 78: 6324-6328.

16. Riley MA and Gordon DM (1999) The ecological role of bacteriocins in bacterial competition. Trends in Microbiology 7: 129-133.

17. Graham AL (2008) Ecological rules governing helminth-microparasite coinfection. Proceedings of the National Academy of Sciences of the United States of America 105: 566-570.

18. Pedersen AB and Fenton A (2007) Emphasizing the ecology in parasite community ecology. Trends in Ecology \& Evolution 22: 133-139.

19. Sibley CD, Duan K, Fischer C, et al. (2008) Discerning the complexity of community interactions using a Drosophila model of polymicrobial infections. PLoS Pathogens 4: e1000184. 
20. Madhi SA, Adrian P, Kuwanda L, et al. (2007) Long term effect of pneumococcal conjugate vaccine on nasopharyngeal colonization by Streptococcus pneumoniae and associated interactions with Staphylococcus aureus and Haemophilus influenzae colonization in HIV-Infected and HIV-uninfected children. Journal of Infectious Diseases 196: 16621666.

21. Madhi SA, Izu A, Nunes MC, et al. (2015) Longitudinal study on Streptococcus pneumoniae, Haemophilus influenzae and Staphylococcus aureus nasopharyngeal colonization in HIV-infected and -uninfected infants vaccinated with pneumococcal conjugate vaccine. Vaccine 33: 2662-2669.

22. Bisgaard H, Hermansen MN, Buchvald F, et al. (2007) Childhood asthma after bacterial colonization of the airway in neonates. New England Journal of Medicine 357: 1487-1495.

23. Graham PL 3rd, Lin SX and Larson EL (2006) A U.S. population-based survey of Staphylococcus aureus colonization. Annals of Internal Medicine 144: 318-325.

24. Davis MF, Peng RD, McCormack MC, et al. (2015) Staphylococcus aureus colonization is associated with wheeze and asthma among US children and young adults. Journal of Allergy and Clinical Immunology 135: 811-813.

25. Sintobin I, Keil T, Lau S, et al. (2015) Is immunoglobulin E to Staphylococcus aureus enterotoxins associated with asthma at 20 years? Pediatric Allergy and Immunology. DOI: 10.1111/pai.12396.

26. Esposito S, Colombo C, Tosco A, et al. (2015) Streptococcus pneumoniae oropharyngeal colonization in children and adolescents with cystic fibrosis. Journal of Cystic Fibrosis. DOI: 10.1016/j. jcf.2015.05.008.

27. Chao Y, Marks LR, Pettigrew MM, et al. (2015) Streptococcus pneumoniae biofilm formation and dispersion during colonization and disease. Frontiers in Cellular and Infection Microbiology 4: 194.

28. Kornum JB, Thomsen RW, Riis A, et al. (2008) Diabetes, glycemic control, and risk of hospitalization with pneumonia: A population-based case-control study. Diabetes Care 31: 1541-1545.

29. Muller LM, Gorter KJ, Hak E, et al. (2005) Increased risk of common infections in patients with type 1 and type 2 diabetes mellitus. Clinical Infectious Diseases 41: 281-288.
30. Seminog OO and Goldacre MJ (2013) Risk of pneumonia and pneumococcal disease in people hospitalized with diabetes mellitus: English record-linkage studies. Diabetic Medicine 30: 1412-1419.

31. Menne EN, Sonabend RY, Mason EO, et al. (2012) Staphylococcus aureus infections in pediatric patients with diabetes mellitus. Journal of Infection 65: 135141.

32. Le Polain de Waroux O, Flasche S, Prieto-Merino D, et al. (2014) Age-dependent prevalence of nasopharyngeal carriage of Streptococcus pneumoniae before conjugate vaccine introduction: A prediction model based on a meta-analysis. PLoS One 9: e86136.

33. Centers for Disease Control and Prevention. Recommended immunizations for children from 7 to 18 years old. Available at: http://www.cdc.gov/vaccines/who/teens/downloads/parent-version-schedule7-18yrs.pdf (accessed 22nd May 2015).

34. Loo JD, Conklin L, Fleming-Dutra KE, et al. (2014) Systematic review of the indirect effect of pneumococcal conjugate vaccine dosing schedules on pneumococcal disease and colonization. Pediatric Infectious Disease Journal 33 Suppl 2: S161-S171.

35. Principi N, Terranova L, Zampiero A, et al. (2015) Pharyngeal colonization by Streptococcus pneumoniae in older children and adolescents in a geographical area characterized by relatively limited pneumococcal vaccination coverage. Pediatric Infectious Disease Journal 34: 426-432.

36. Pericone CD, Overweg K, Hermans PW, et al. (2000) Inhibitory and bactericidal effects of hydrogen peroxide production by Streptococcus pneumoniae on other inhabitants of the upper respiratory tract. Infection and Immunity 68: 3990-3997.

37. Lijek RS, Luque SL, Liu Q, et al. (2012) Protection from the acquisition of Staphylococcus aureus nasal carriage by cross-reactive antibody to a pneumococcal dehydrogenase. Proceedings of the National Academy of Sciences of the United States of America 109: 13823-13828.

38. Bosch AA, Biesbroek G, Trzcinski K, et al. (2013) Viral and bacterial interactions in the upper respiratory tract. PLoS Pathogens 9: e1003057.

39. Regev-Yochay G, Lipsitch M, Basset A, et al. (2005) The pneumococcal pilus predicts the absence of Staphylococcus aureus co-colonization in pneumococcal carriers. Clinical Infectious Diseases 48:760-763. 